\title{
Enhanced exponential rule scheduling algorithm for real-time traffic in LTE network
}

\author{
J. I. A.Y. Yaqoob, W. L. Pang, S. K. Wong, K. Y. Chan \\ Faculty of Engineering, Multimedia University, Malaysia
}

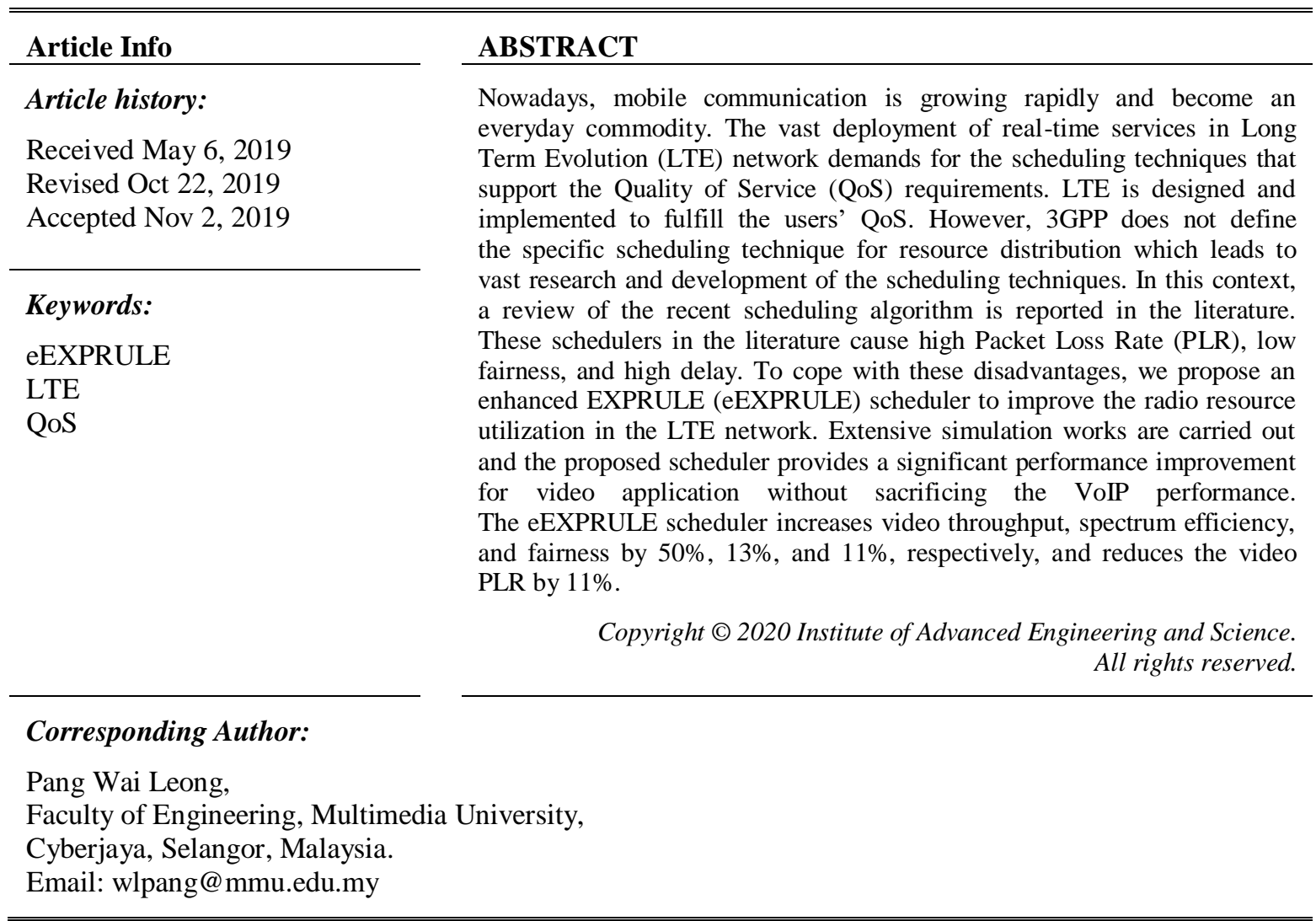

\section{INTRODUCTION}

In conjunction with the continuing rise of multimedia, File Transfer Protocol (FTP) and non-realtime (NRT) services, the LTE wireless standard have emerged to efficient resource management [1]. The main features of LTE are low delay, high data rate as well as better packet optimization. One of the key features of LTE is the Radio Resource Management procedure which raises the system performance up to the Shannon limit [2]. Packet scheduling mechanisms, in particular, are a corner stone of LTE, since they are responsible for choosing, with proper time and frequency resolutions, how to distribute the available radio resources among the stations with taking into account the channel condition and QoS requirements [3]. For the QoS requirements for real-time (RT) and NRT are varied in term of delay and data rate, it is necessary to develop an efficient scheduling technique to achieve the QoS requirements [4-6]. Third Generation Partnership Project (3GPP) is the main body that developed LTE, however, it does not define the unified scheduling algorithm. For this reason, scheduling development has been given vast research concern [3]. In order to satisfy various QoS requirements in LTE, a trade-off between the fairness, packet loss rate, and average throughput must be accomplished. There are two main traffic categories: RT and NRT, which demand different QoS. 3GPP categories them as guarantee bit rate (GBR) and non-grantee bit rate (non-GBR). Video and Voice over IP (VoIP) traffics are in RT category. Constant bit rate and best-effort traffics are categorized as NTR traffics [7]. When it comes to QoS in LTE downlink system, there are a variety of factors that influence the system performance, including users' channel quality 
and resource allocation technique. In LTE, the transmission resource is divided into frames, which each frame contains 10 sub-frames. Each sub-frame can be segmented into two slots which each slot is equal to $0.5 \mathrm{~ms}$ as depicted in figure 1. One of the features that implemented in LTE is the flexible channel bandwidth and there are a variety of channels with different bandwidth. The number of physical resource blocks (RB) depends on the channel bandwidth. In general, a resource block is composed of 12 subcarriers whose bandwidth is equal to $15 \mathrm{kHz}$. Each RB is equal to a slot duration as illustrated in Figure 1 [8-14].

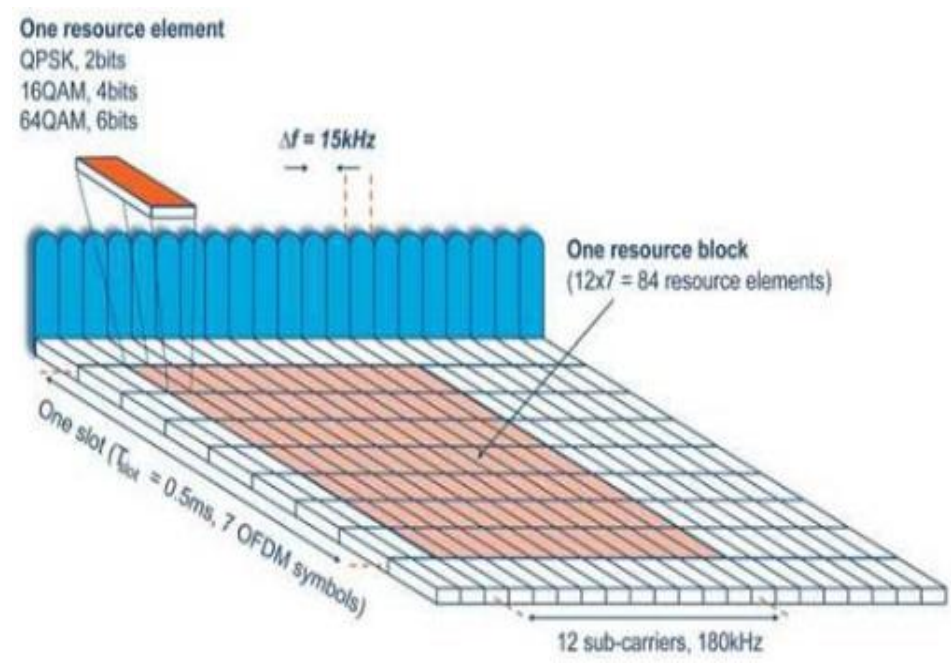

Figure 1. LTE frame structure [9]

When it comes to the resource allocation using QoS scheduling, there are several factors that affecting the distribution mechanism such as the number of RBs available, the number of active users, the size of flow buffer, the average past throughput, the maximum flow delay, the Packet Lose Rate (PLR), and the Channel Quality Indicator (CQI) which periodically reported by User Equipment (UEs) [9] to the base station. The base station of LTE, Evolved NodeB (eNodeB) is responsible for resource allocation determination at its Medium Access Control (MAC) layer [14]. The distribution is different corresponding to the different scheduling mechanisms.

The scheduler in the LTE system is a sequence process that calculates a metric for each application and to allocate the resources effectively by comparing the metric value of each UE among the existing UEs. The $i$-th UE will be allocated the $j$-th RB if the $i$-th UE has the maximum metric value. The RB allocation is decided by this metric, which based on different QoS needs. The metric value is usually computed from the relevant information, for instance, the number of RBs available, the size of flow buffer and delay threshold, the average past throughput and PLR values [15].

\section{LTE SCHEDULERS}

The following are the description of the LTE schedulers that used in simulation which are Proportional Fair (PF), Modified Largest Waited Delay First (MLWDF), Exponential PF (EXP/PF), Exponential Rule (EXPRULE), Logarithm Rule (LOGRULE), and the proposed scheduler eEXPRULE.

\subsection{Proportional fair (PF) scheduler}

The PF scheduler provides the tradeoff between the system throughput and fairness by taking into account the ratio between the instantaneous flows available for i-th flow and the average flow rate. This scheduler is suitable for non-real time traffic [16]. The transmission metric for this scheduler is as follows:

$$
\omega_{i, j}=\frac{r_{i, j}}{\bar{R}_{i}}
$$

Where $r_{i, j}$ is the rate assigned to $\mathrm{i}$-th flow during the k-th Transmission Time Interval (TTI) and $\bar{R}_{i}$ denotes the average estimated data rate. 


\subsection{Modified largest weighted delay first (MLWDF) scheduler}

The MLWDF scheduler is designed to support mixed RT and NRT traffics. It calculates the transmission metric by taking into account the instantaneous channel variation and head-of-line delay [17]. The equation is shown as follows:

$$
\omega_{i, j}=\alpha_{i} D_{H o L, i} \frac{r_{i, j}}{\overline{R_{i}}}
$$

where $\omega_{\mathrm{i}, \mathrm{j}}$ is the transmission metric for RT applications, $\mathrm{D}_{\mathrm{HoL}, \mathrm{i}}$ is the head-of-line delay of $\mathrm{i}$-th $\mathrm{UE}, r_{i, j}$ is the rate assigned to $\mathrm{i}$-th flow during the $\mathrm{k}$-th TTI, $\bar{R}_{i}$ denotes the average estimated data rate, and $\alpha_{i}$ is given in (3).

$$
\alpha_{i}=-\frac{\log \delta_{i}}{\tau_{i}}
$$

where $\tau_{i}$ is the $\mathrm{i}$-th user delay threshold.

\subsection{Exponential PF (EXP/PF) scheduler}

The EXP/PF scheduler was developed to support multiple types of traffic by giving priority to RT traffic by computing the transmission metric using equation 4 while for NRT traffic use PF scheduler [18].

$$
\omega_{i, j}=\exp \left(\frac{\alpha_{i} D_{H o L, i}-X}{1+\sqrt{X}}\right) \frac{r_{i, j}}{\overline{R_{i}}}
$$

where $\mathrm{X}$ is given as:

$$
\mathrm{X}=\frac{1}{\mathrm{~N}_{\mathrm{rt}}} \sum_{\mathrm{i}=1}^{\mathrm{N}_{\mathrm{rt}}} \alpha_{\mathrm{i}} \mathrm{D}_{\mathrm{HoL}, \mathrm{i}}
$$

Where $N_{r t}$ denotes the number of active UEs for RT traffics.

\subsection{Exponential rule (EXPRULE) scheduler}

This scheduler takes into account the head-of-line packet delay and CQI, which frequently reported by UEs, to support the delay sensitive traffic such as VoIP [19]. The transmission metric is computed based on equation 6 which improved in term of delay and throughput compared to EXP/PF [3].

$$
\omega_{i, j}=b_{i} \exp \left(\frac{\alpha_{i} D_{H o L, i}}{c+\sqrt{\left(\frac{1}{N_{r t}}\right) \sum D_{H o L, i}}}\right) \Gamma_{k}^{i}
$$

where $\Gamma_{k}^{i}$ is the spectrum efficiency for i-th user on k-th sub-channel. $\alpha_{i}$ and $b_{i}$ are given as follows, which $\mathrm{c}$ is equaled to 1 :

$$
\begin{aligned}
& \alpha_{i} \in\left[\frac{5}{\left(0.99 \tau_{i}\right)}, \frac{10}{\left(0.99 \tau_{i}\right)}\right] \\
& b_{i}=\frac{1}{E\left[\Gamma^{i}\right]}
\end{aligned}
$$

\subsection{Logarithm rule (LOGRULE) scheduler}

The LOGRULE scheduler aims to contribute an efficient QoS support to the network by maintaining the average delay in the system. The transmission metric in some degree is identical to the EXPRULE scheduler, the variance is that this scheduler impalement the logarithm function approach which is the inverse of exponential function [20]. The scheduler for NRT transmission uses the PF scheduler and for the RT the transmission metric is calculated based on (9).

$$
\omega_{i, j}=b_{i} \cdot \ln \left(c+\alpha_{i} D_{H o L, i}\right) \Gamma_{k}^{i}
$$




\subsection{Enhanced exponential rule (eEXPRULE) scheduler}

The proposed eEXPRULE try to reduce the head-of-line delay by computing the transmission metric for each traffic separately, which leads to an increment in the UE's throughput. The transmission metric for each type of traffic are as follows:

Transmission metric for best effort traffic:

$$
\omega_{i, j}=\frac{r_{i, j}}{\bar{R}_{i}}
$$

transmission metric for VoIP traffic:

$$
\omega_{i, j}=\exp \left(\frac{\left(\alpha_{i} D_{H o L, i}\right)}{c+\sqrt{\left(\frac{1}{N_{r t}}\right) \sum D_{H o L, i}}} \frac{r_{i, j}}{\bar{R}_{i}}\right)
$$

transmission metric for video traffic:

$$
\omega_{i, j}=\exp \left(\frac{\left(\alpha_{i} D_{H o L, i}\right)}{c+\sqrt{\left(\frac{1}{N_{r t}}\right) \sum D_{H o L, i}}}\right) r_{i, j}
$$

where $\alpha_{i}$ is given as follow, in which $c$ is equaled to 1 :

$$
\alpha_{i} \in\left[\frac{5}{\left(\tau_{I}\right)}, \frac{10}{\left(\tau_{I}\right)}\right]
$$

the proposed eEXPRULE algorithm.

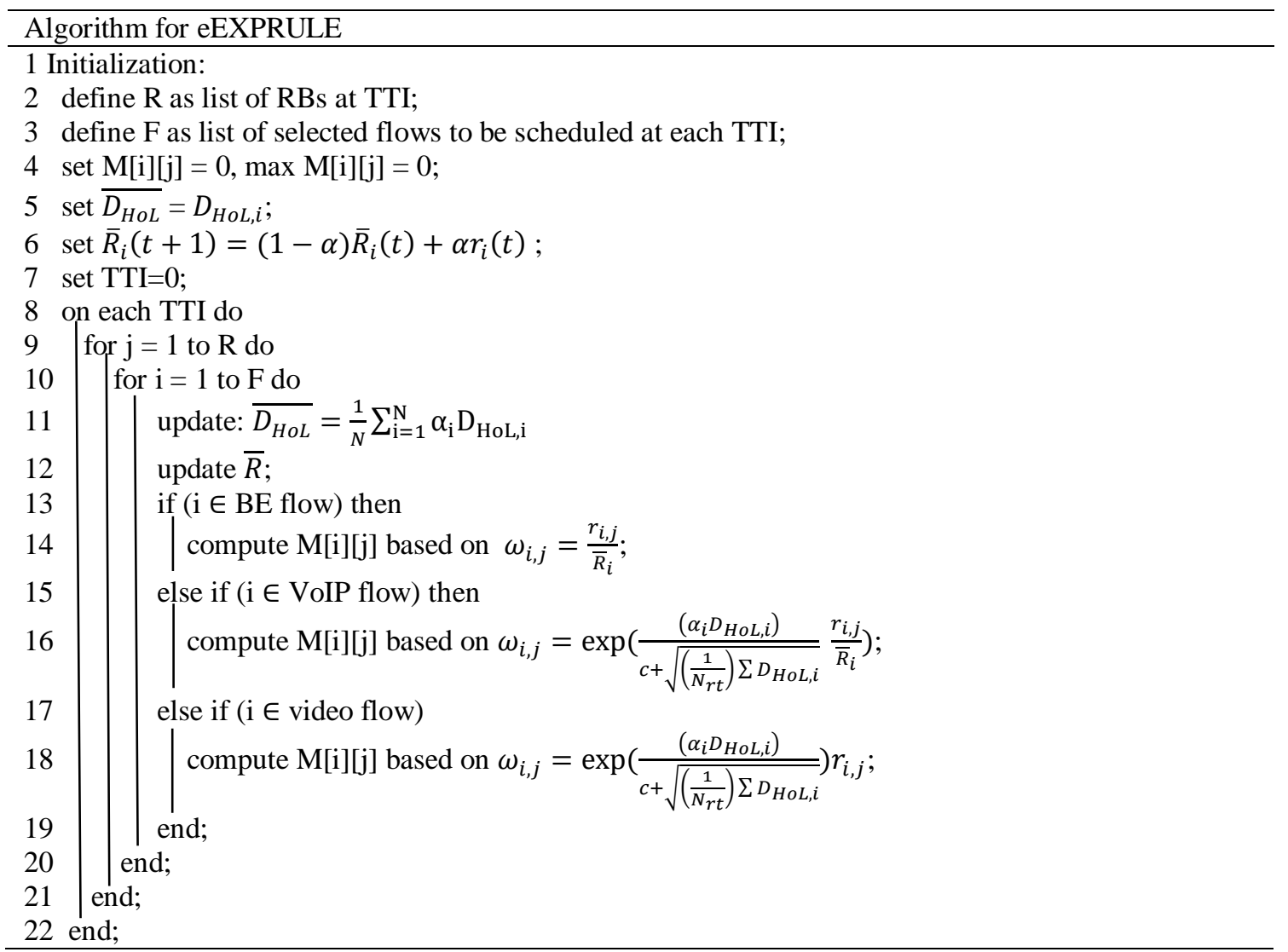




\section{RESULTS AND DISCUSSIONS}

This paper studies the relevant performance of the aforementioned schedulers as well as the proposed scheduler. The simulation is carried out in a single cell scenario without interference. Multiple UEs which set to the free motion model with random position within the cell and connected to an eNodeB. The simulation works are carried out using the open source simulator LTE-Sim [15]. Table 1 summarizes the simulation parameters used in the simulation.

Table 1.. Simulation parameters

\begin{tabular}{llll}
\hline Parameters & Values & Parameters & Values \\
\hline Simulation duration & $100 \mathrm{~s}$ & Number of UEs & 100 \\
Flow duration & 15 & Bandwidth & $10 \mathrm{MHz}$ \\
Frame structure & FDD & Number of RBs & 50 \\
Cell radius & $1 \mathrm{~km}$ & Maximum delay & $0.1 \mathrm{~s}$ \\
UE speed & $3 \mathrm{~km} / \mathrm{h}$, Random Direction & Video bit-rate & $242 \mathrm{kbps}$ \\
\hline
\end{tabular}

\subsection{Traffic model}

In this simulation, there is a single eNodeB in a single cell that communicates with UEs in $10 \mathrm{MHz}$ bandwidth. One video, one VoIP and one best effort flow are carried out in parallel by each UE. For the video flow, a trace-based application that emits the packets based on a realistic video trace file with a rate of $242 \mathrm{kbps}$ is used. For VoIP, a G.729 voice stream with a maximum delay of $0.1 \mathrm{~s}$ is used [21]. G.729 is an audio codec with the lowest bit rate that squeezes digital conversation in the packets of $10 \mathrm{~ms}$ length. Due to the low bit rate it presents the chances for important augment in bandwidth consumption in the existing technology [22]. The voice flow is a burst application that is modelled with an ON/OFF Markov chain [15]. We run the simulation with multiple UEs in the range of 10 to 100 UEs with a step increment of 10 using the Frequency Division Duplex (FDD) mode.

\subsection{Performance measurement}

The performance evaluation of the schedulers is based on throughput, fairness, spectrum efficiency, and delay. Throughput is calculated as the total number of bits that successfully transmitted from eNodeB to UE over the total simulation time [23].

$$
\text { Throughput }=\frac{\text { total data received by } U E}{\text { simulation time }}
$$

The spectrum efficiency is calculated based on equation 15 :

$$
\text { Spectrum efficiency }=\frac{\text { goodput } / \text { time }}{\text { bandwidth }}
$$

One of the key factors to evaluate the performance of the scheduler is fairness. Fairness is a measurement of the equal distribution of the system resources among the UEs [24]. We used the Jain Fairness Index to measure the fairness of the schedulers [25]. It states that if a system allocates a resource to $n$ users with its share its $i$-th throughput $\left(T_{i}\right)$ than the fairness index can be calculated as:

$$
\text { Jain Fairness Index }=\frac{\left[\sum_{i=1}^{n} T_{i}\right]^{2}}{n \sum_{i=1}^{n} T_{i}^{2}}
$$

\subsection{Simulation results}

In this section, the simulation results are highlighted in order to evaluate the performance of the proposed scheduler using LTE-Sim open source simulator. The simulator supports the single and multicell environments, multi-user, and QoS management, which is developed based on 3GPP specifications [26]. The performance analyses are mainly focused on the RT traffic, i.e. video and VoIP. In order to evaluate the performance of eEXPRULE, we compared the performance of eEXPRULE with the second-best scheduler at 100 UEs. The eEXPRULE separates each traffic type of metric computation which leads to the increment of the spectrum efficiency by 13\% compared to the LOGRULE, MLWDF, and EXP/PF which have the same and higher spectrum efficiency among the existing schedulers as it can be seen in Figure 2. Compare to the existing schedulers which they categorize the traffic to the RT and NRT traffic. The spectrum efficiency of eEXPRULE increases as the number of UEs increases while in the other schedulers they tend to decrease. 


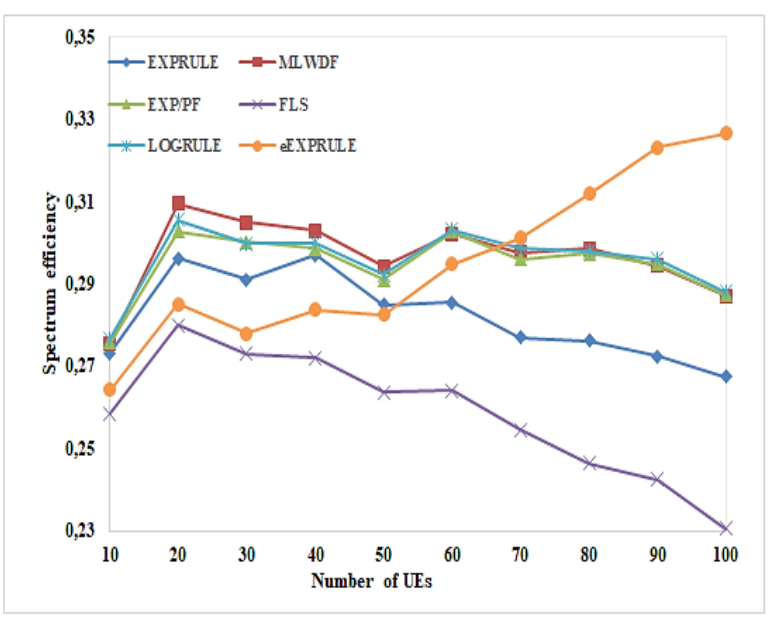

Figure 2. Spectrum efficiency

As it mentioned in the literature, in order to fulfill the QoS required by each user, the scheduler should provide an optimal trade-off between fairness and spectrum efficiency, which can be further extended to throughput and delay. Figure 3(a) illustrates the video fairness index, which calculated based on Jain's fairness index as formulated in (16). Since in the proposed scheduler, we separate the traffic metric calculation, the fairness improved by $11 \%$ with respect to the FLS scheduler which has the highest fairness in this application. Figure 3(a) depicts that the proposed scheduler has a lower drop rate among the exciting schedulers as the number of UEs increase. The VoIP fairness is illustrated in Figure 3(b) which is maintained at 0.32 for all schedulers when the number of UEs is more than 40 .

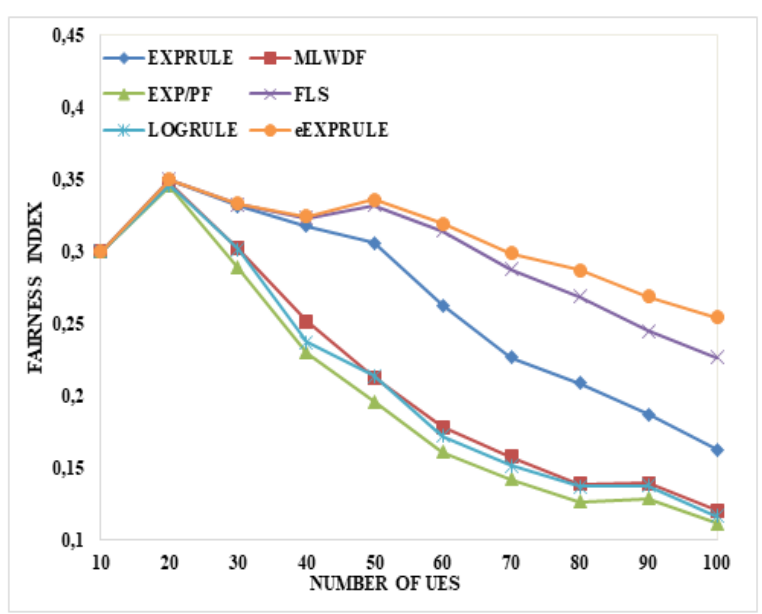

(a)

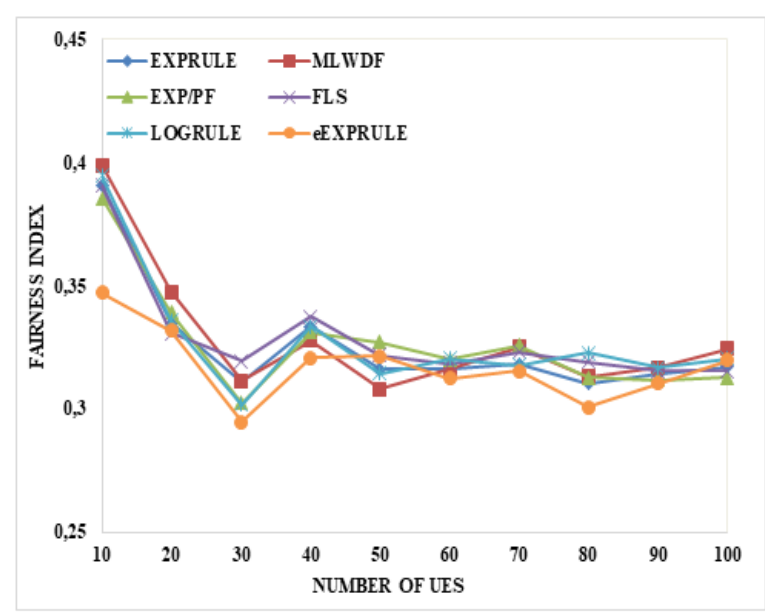

(b)

Figure 3. (a) Video fairness index; (b) VoIP fairness index

For the RT traffic, the Packet Loss Rate (PLR) is a critical factor for QoS satisfactory. PLR depends on the scheduler delay threshold; which means a higher delay threshold gives a lower PLR. As shown in Figure 4(a), the video PLR values increased once the number of UEs increased that contributed to the long packet queuing time. The eEXPRULE has outperformed over the existing schedulers by $11 \%$ improvement for video application. The eEXPRULE has 40\% PLR for 100 UEs in a video application. Figure 4(b) illustrates the PLR for VoIP application which is highly fluctuating. This ripple is about $1 \%$ for the worst scenario. The proposed scheduler has less than $0.2 \%$ PLR for all UEs. 


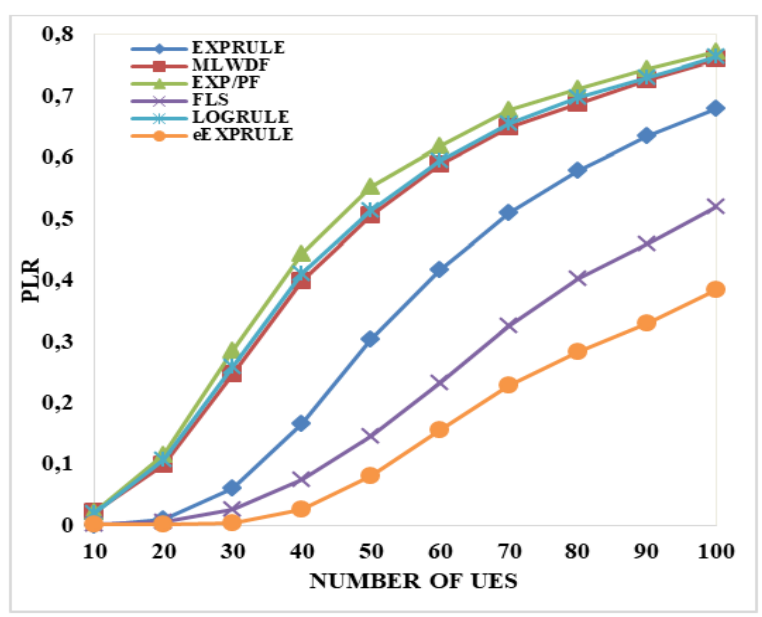

(a)

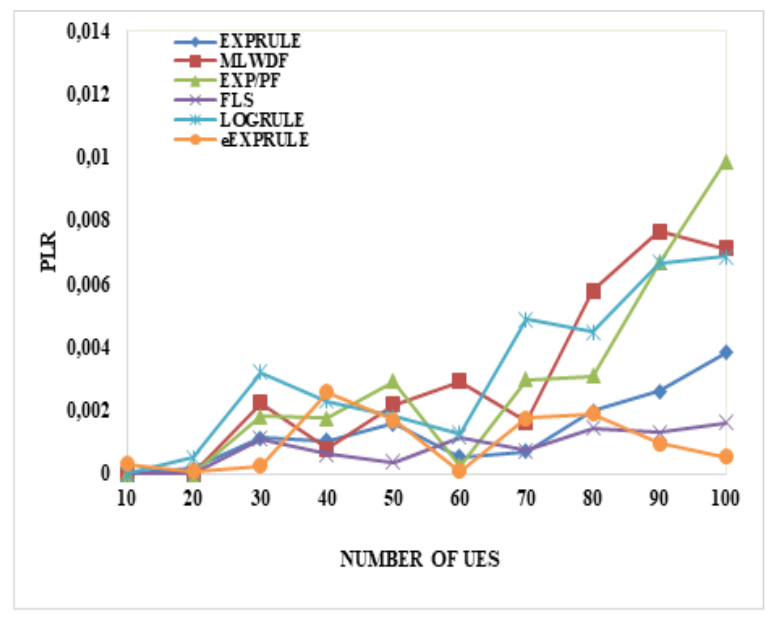

(b)

Figure 4. (a) Video PLR; (b) VoIP PLR

Figure 5(a) depicts video performance throughput with respect to the number of UEs. The proposed scheduler significantly outperforms over the existing schedulers which only consider the instantaneous data rate in the transmission metric. It has 50\% improvement compared to the FLS scheduler for 100 UEs. The video throughput of eEXPRULE is gradually increasing as the number of UEs increasing. This increment can be related to the improvement of the spectrum efficiency in the proposed scheduler as it is depicted in Figure 2 while the video throughput of the exciting schedulers tends to decrease gradually. Figure 5(b) depicts all schedulers have the same performance for the VoIP application, which can be understood that eEXPRULE improved the video application performance and maintained the VoIP application performance.

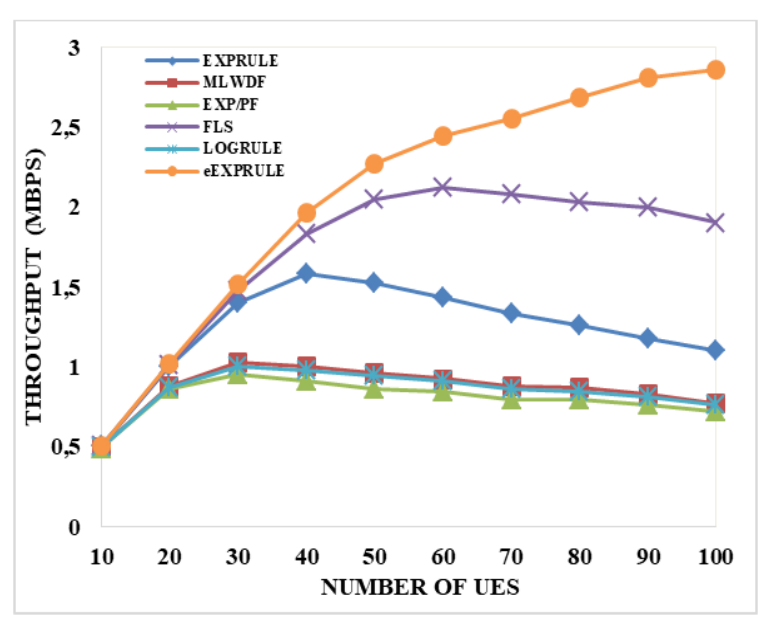

(a)

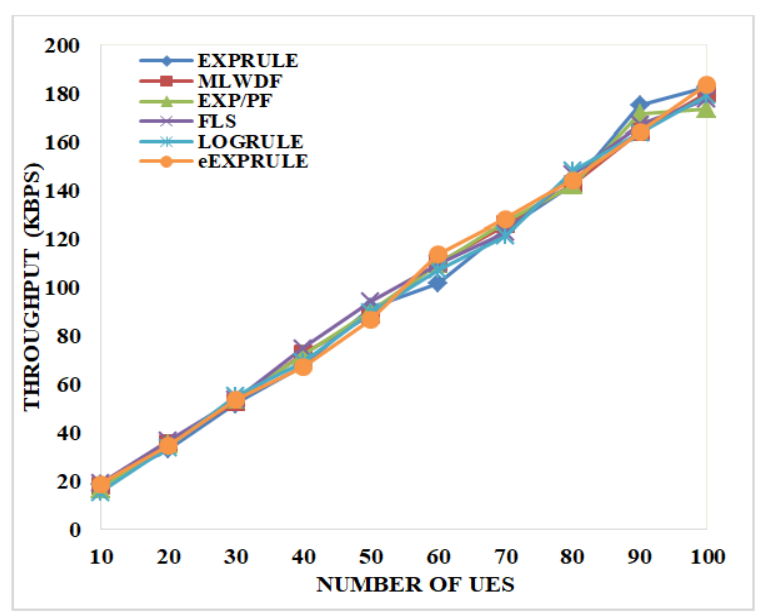

(b)

Figure 5. (a) Video throughput; (b) VoIP throughput

As shown in Figure 6(a), the eEXPRULE has a lower video delay for the number of UEs less than 40. However, it grows gradually to 0.07 second for 100 UEs. Figure 6(b) illustrates the VoIP delay, which is a critical factor in this specific application. In the proposed scheduler, we interrelate the delay factor with the channel data rate as formulated in (12). As the FLS and eEXPRULE have a constant delay while the eEXPRULE outperforms over FLS scheduler. 


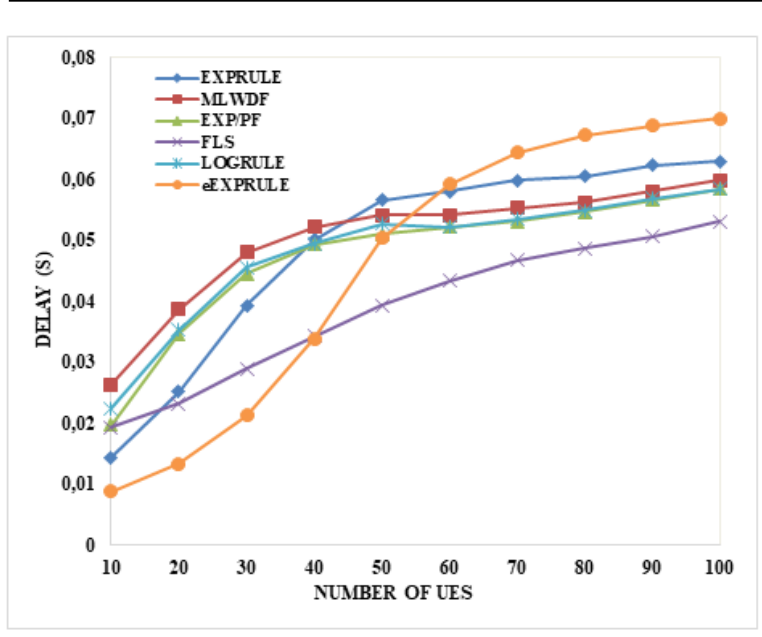

(a)

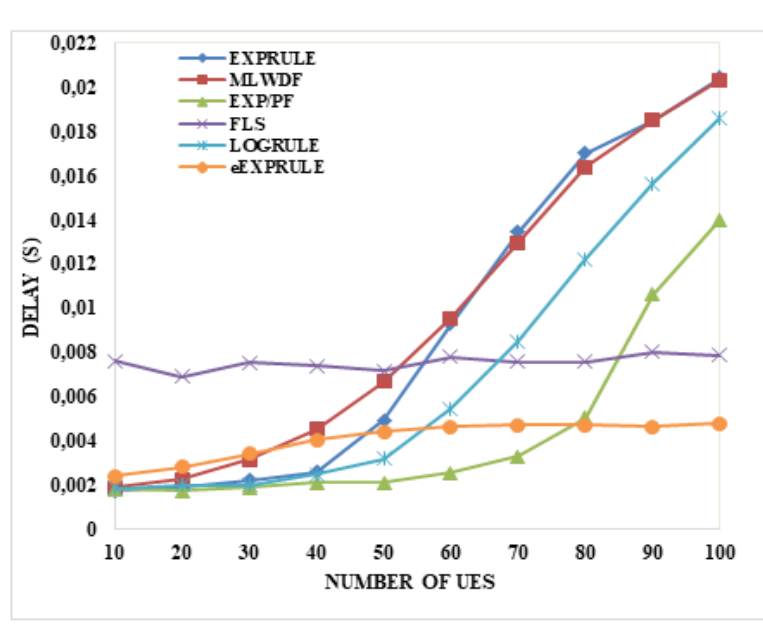

(b)

Figure 6. (a) Video delay. (b) VoIP delay

\section{CONCLUSION}

This paper studies the performance of the existing schedulers, EXPRULE, EXP/PF, MLDWF, FLS, and LOGRULE using LTE-Sim simulator as well as proposing an enhanced scheduler, eEXPRULE for RT traffic. The proposed scheduler is the enhanced EXPRULE scheduler. It separates each traffic metric computation based on the critical factors in each traffic flow. Extensive simulations are carried out and the results are promising that indicate the eEXPRULE has better performance over the existing schedulers as well as EXPRULE. The proposed eEXPRULE improves the video traffic performance in term of throughput, PLR, and fairness by $50 \%, 11 \%, 11 \%$ respectively while the VoIP performance maintained.

\section{ACKNOWLEDGEMENTS}

This work was supported in part by TM Research and Development under Grant MMUE/170013.

\section{REFERENCES}

[1] D. Samia and B. Ridha, "A New Scheduling Algorithm for Real-Time Communication in LTE Networks," Proc. IEEE 29th Int. Conf. Adv. Inf. Netw. Appl. Work. WAINA 2015, pp. 267-271, 2015.

[2] Y. Gao et al., "A novel resource allocation in imperfect D2D cooperation in LTE-Advanced Pro systems," 7th IEEE Annu. Inf. Technol. Electron. Mob. Commun. Conf. IEEE IEMCON 2016, pp. 1-5, 2016.

[3] M. Iturralde, T. Ali Yahiya, A. Wei, and A. L. Beylot, "Performance study of multimedia services using virtual token mechanism for resource allocation in LTE networks," IEEE Veh. Technol. Conf., pp. 1-5, 2011.

[4] Y. P. Li, B. J. Hu, H. Zhu, Z. H. Wei, and W. Gao, "A delay priority scheduling algorithm for downlink real-time traffic in LTE networks," Proc. 2016 IEEE Inf. Technol. Networking, Electron. Autom. Control Conf. ITNEC 2016, pp. 706-709, 2016.

[5] E. Skondras, A. Michalas, A. Sgora, and D. D. Vergados, "A downlink scheduler supporting real time services in LTE cellular networks," in 2015 6th International Conference on Information, Intelligence, Systems and Applications (IISA), 2015, pp. 1-6.

[6] N. K. M. Madi, Z. M. Hanapi, M. Othman, and S. K. Subramaniam, "Delay-based and QoS-aware packet scheduling for RT and NRT multimedia services in LTE downlink systems," Eurasip J. Wirel. Commun. Netw., vol. 2018, no. 1, 2018.

[7] X. Chen, Y. Liu, and Y. Wang, "A Novel Downlink Scheduler Based on Q-Learning for Video Traffic in LTE Networks," Proc. 2018 6th IEEE Int. Conf. Netw. Infrastruct. Digit. Content, IC-NIDC 2018, vol. 7, pp. 115-119, 2018.

[8] M. Mamman, Z. M. Hanapi, A. Abdullah, and A. Muhammed, "Quality of service class identifier (QCI) radio resource allocation algorithm for LTE downlink," PLoS One, vol. 14, no. 1, pp. 1-22, 2019.

[9] N. N. Sirhan, G. L. Heileman, C. C. Lamb, and R. Piro-Rael, "QOS-Based Performance Evaluation of ChannelAware/QOS-Aware Scheduling Algorithms for Video Applications Over LTE/LTE-A," in Computer Science \& Information Technology ( CS \& IT ), 2015, pp. 49-65.

[10] N. K. M. Madi, Z. B. M. Hanapi, M. Othman, and S. Subramaniam, "Two-level QoS-aware frame-based downlink resources allocation for RT/NRT services fairness in LTE networks," Telecommun. Syst., vol. 66, no. 3, 
pp. 357-375, 2017.

[11] S. Alotaibi and R. Akl, "Radio resource management in LTE femtocell networks," in 2017 IEEE 16th International Symposium on Network Computing and Applications (NCA), 2017, pp. 1-5.

[12] T. Sheu, K. Chang, and F. Yeh, "A Dynamic Allocation Scheme for Resource Blocks Using ARQ Status Reports in LTE Networks," 2017 Int. Conf. Information, Commun. Eng., pp. 224-227, 2017.

[13] S. Liu, C. Zhang, Y. Zhou, and Y. Zhang, "Delay-Based Weighted Proportional Fair Algorithm for LTE Downlink Packet Scheduling,” Wirel. Pers. Commun., vol. 82, no. 3, pp. 1955-1965, Jun. 2015.

[14] E. Dahlman, S. Parkvall, and J. Skold, 4G LTE-advanced pro and the road to 5G. Elsevier, 2016.

[15] G. Piro, L. A. Grieco, G. Boggia, F. Capozzi, and P. Camarda, "Simulating LTE Cellular Systems: An OpenSource Framework," IEEE Trans. Veh. Technol., vol. 60, no. 2, pp. 498-513, Feb. 2011.

[16] J.-G. Choi and S. Bahk, "Cell-Throughput Analysis of the Proportional Fair Scheduler in the Single-Cell Environment," IEEE Trans. Veh. Technol., vol. 56, no. 2, pp. 766-778, Mar. 2007.

[17] P. Ameigeiras, J. Wigard, and P. Mogensen, "Performance of the M-LWDF scheduling algorithm for streaming services in hsdpa," in IEEE 60th Vehicular Technology Conference, 2004. VTC2004-Fall. vol. 2, pp. 999-1003, 2004.

[18] F. Afroz, K. Sandrasegaran, and P. Ghosal, "Performance analysis of PF, M-LWDF and EXP/PF packet scheduling algorithms in 3GPP LTE downlink," in 2014 Australasian Telecommunication Networks and Applications Conference (ATNAC), 2014, pp. 87-92.

[19] E. M. Ang, K. K. Wee, Y. H. Pang, and S. H. Lau, "Two-Level Scheduling Framework with Frame Level Scheduling and Exponential Rule in Wireless Network," in 2014 International Conference on Information Science \& Applications (ICISA), 2014, pp. 1-4.

[20] E. M. Ang, K. K. Wee, Y. H. Pang, and K. K. Phang, "A performance analysis on packet scheduling schemes based on an exponential rule for real-time traffic in LTE," Eurasip J. Wirel. Commun. Netw., vol. 2015, no. 1, pp. 1-12, 2015.

[21] A. M. Al-Dulaimi, E. M. Al-Azzawi, and A. I. Al-Anssari, "Balancing model of resource blocks allocation in LTE downlink," in 2016 International Conference on Electronics and Information Technology (EIT), 2016, pp. 1-4.

[22] R. C. Soothar, M. Pathan, B. Qureshi, P. K. Butt, and G. Mujtaba, "Analysis of Voip Traffic Service in 4G Lte Cellular Networks,” Indian J. Sci. Technol., vol. 11, no. 16, pp. 1-6, 2018.

[23] A. A. A. Rahman, N. M. Sharipp, A. K. Samingan, and C. Y. Yeoh, "Improved link level LTE scheduler," in 2015 10th International Conference on Information, Communications and Signal Processing (ICICS), 2015, pp. 1-5.

[24] I. I. Al-Shiab and R. E. Ahmed, "On fairness in LTE downlink MAC scheduling algorithms," in 2015 International Conference on Information and Communication Technology Research (ICTRC), 2015, pp. 171-174.

[25] R. Jain, D. Chiu, and W. Hawe, "A Quantitative Measure Of Fairness And Discrimination For Resource Allocation In Shared Computer Systems,” Digit. Equip. Corp. Res. Rep., 1984.

[26] A. Pellegrini and G. Piro, "Multi-threaded simulation of $4 \mathrm{~g}$ cellular systems within the LTE-sim framework," Proc. - 27th Int. Conf. Adv. Inf. Netw. Appl. Work. WAINA 2013, pp. 101-106, 2013.

\section{BIOGRAPHIES OF AUTHORS}

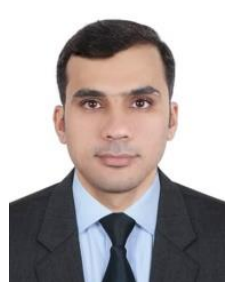

Yaqoob J. I. A. Yghoubi received B.E degree in Electronics from Multimedia University, Malaysia in March 2017. Currently a M.Eng.Sc student at Multimedia University, Malaysia. His research interests focus on the media access control, scheduling algorithms and quality of service in the OFDMA-based wireless network.

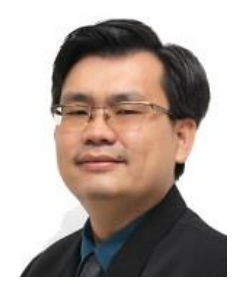

Wai-Leong Pang received his Ph.D. degree in 2013. He is a Professional Engineer with Practising Certificate registered with the Board of Engineers Malaysia, a corporate member with The Institution of Engineers Malaysia and a senior member with IEEE. His research interests include wireless communications, networking, VLSI, digital design and renewable energy. Since 2004, he has been with Multimedia University, Malaysia. Currently, he is the Programme Coordinator of the B.Eng. (Hons) Electronics under Faculty of Engineering, Multimedia University, Malaysia.

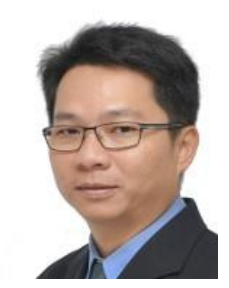

Sew-Kin Wong received his B.Eng degree in Electrical and Electronic Engineering in 1995. He then obtained his M.Eng.Sc degree and Ph.D degree in 2003 and 2011 respectively. His research interests are RF transceiver and RFIC design for the next generation wireless systems and EMI/EMC compliances and shielding techniques. He is currently a member of the Faculty of Engineering, Multimedia University, Malaysia. 
Kah-Yoong Chan obtained his Ph.D. in Electrical Engineering from Jacobs University Bremen in Germany in year 2008. He is a Professional Engineer with Practising Certificate (PEPC) with BEM, a senior member with IEEE, and a corporate member with IEM. Ir. Dr. Chan research areas are in the micro and nanoelectronic device fabrications and characterizations. Currently, he is an Associate Professor with Faculty of Engineering in Multimedia University, and serving as a chaiperson for a Research Center under Faculty of Engineering of MMU - Centre for Advanced Devices and Systems (CADS). 